Ciência Florestal, Santa Maria, v. 23, n. 4, p. 761-769, out.-dez., 2013

ISSN 0103-9954

\title{
PROPRIEDADES FÍSICAS E MECÂNICAS DE PAINÉIS DE MADEIRA AGLOMERADA DE Acrocarpus fraxinifolius, COMPOSTOS COM DIFERENTES PERCENTUAIS DE CASCA
}

\author{
PHYSICAL AND MECHANICAL PROPERTIES OF PARTICLEBOARDS OF Acrocarpus fraxinifolius \\ COMPOUNDS WITH DIFFERENT PERCENTAGES OF BARK
}

\author{
Rosilani Trianoski ${ }^{1}$ Setsuo Iwakiri ${ }^{2}$ Jorge Luis Monteiro de Matos ${ }^{3}$ José Guilherme Prata ${ }^{4}$
}

\section{RESUMO}

A indústria de painéis de madeira aglomerada tem utilizado grandes volumes de madeira, sobretudo de florestas plantadas de Pinus, sendo necessário diversificar espécies, bem como maximizar o uso da matéria-prima. Uma das maneiras de maximizar o uso deste recurso é a utilização da casca no processo produtivo. Desta forma, este trabalho teve como objetivo avaliar as propriedades físicas e mecânicas de painéis aglomerados de Acrocarpus fraxinifolius contendo diferentes percentuais de casca. Foram manufaturados painéis com $100 \%$ de partículas de madeira de Acrocarpus fraxinifolius e painéis com partículas da madeira de Acrocarpus e percentuais de casca de $10 \%, 20 \%$ e 30\%, massa especifica nominal de $0,75 \mathrm{~g} / \mathrm{cm}^{3}$ e $8 \%$ de resina. As propriedades de massa especifica aparente, absorção de água e inchamento em espessura, flexão estática, tração perpendicular à superfície e arrancamento de parafuso foram determinadas de acordo com as normas EN e NBR, e comparadas com um tratamento testemunha (100\% Pinus taeda) e com requisitos normativos prescritos pelas respectivas normas. Os resultados demonstraram que a espécie apresenta viabilidade técnica para produção de painéis de madeira aglomerada e que a crescente adição de casca não afetou negativamente as propriedades físicas e mecânicas dos painéis.

Palavras-chave: painéis de madeira; painéis de madeira aglomerada; Acrocarpus fraxinifolius; casca.

\section{ABSTRACT}

The particleboard industries have used a large volume of wood mainly from planted forest of Pinus, being necessary to diversify the wood species and optimize the use of raw material. One of the ways to optimize the use of this resource is the utilization of the bark in the particleboard manufacturing process. In this way, this research was developed to evaluate the physical and mechanical properties of the particleboard manufactured with the wood of Acrocarpus fraxinifolius and different proportions of the bark. The boards were manufactured with $100 \%$ of wood particles of Acrocarpus fraxinifolius and replacing the wood particles with $30 \%, 20 \%$ and $10 \%$ of bark, board density of $0.75 \mathrm{~g} / \mathrm{cm}^{3}$ and $8 \%$ of urea-formaldehyde resin. The properties of board density, water absorption and thickness swelling, static bending, internal bond and screw pullout strength, were determined according to EN and NBR standards, and then compared with the standard treatment (100\% Pinus taeda) and with the minimum requirements of these standards. The results indicated that Acrocarpus fraxinifolius species showed technical feasibility for particleboard production

1 Engenheira Industrial Madeireira, Dra ${ }^{\mathrm{a}}$, Professora Adjunta do Departamento de Engenharia e Tecnologia Florestal, Universidade Federal do Paraná, Av. Lothário Meissner, 632, Jardim Botânico, Campus III, CEP 80210-170, Curitiba (PR), Brasil. rosilani@ufpr.br

2 Engenheiro Florestal, Dr., Professor Titular do Departamento de Engenharia Florestal e Tecnologia, Universidade Federal do Paraná, Av. Lothário Meissner, 632, Jardim Botânico, Campus III, CEP 80210-170, Curitiba (PR), Brasil. setsuo@ufpr.br

3 Engenheiro Florestal, Dr., Professor Associado do Departamento de Engenharia Florestal e Tecnologia, Universidade Federal do Paraná, Av. Lothário Meissner, 632, Jardim Botânico, Campus III, CEP 80210-170, Curitiba (PR), Brasil.jmatos@ufpr.br

4 Engenheiro Florestal, Dr., Professor Adjunto do Departamento de Engenharia Florestal e Tecnologia, Universidade Federal do Paraná, Av. Lothário Meissner, 632, Jardim Botânico, Campus III, CEP 80210-170, Curitiba (PR), Brasil. prata@ufpr.br

Recebido para publicação em 08/12/2010 e aceito em 14/08/2012 
and the addition of the bark until $30 \%$ did not negatively affect the physical and mechanical properties of the boards.

Keywords: wood panels; particleboard; Acrocarpus fraxinifolius; bark.

\section{INTRODUÇÃO}

O desenvolvimento de painéis de partículas tem revolucionado a industrialização da madeira ao aumentar consideravelmente o rendimento da matéria-prima (VIGNOTE; JIMÉNEZ, 1996). Os painéis de madeira aglomerada são um dos produtos particulados que merecem atenção especial, tendo em vista o aproveitamento integral da madeira, as características isotrópicas do produto e o grande volume de produção, refletindo desta forma, a importância deste segmento na indústria de base florestal (IWAKIRI et al., 2000).

Atualmente, florestas plantadas de Pinus são a principal fonte de matéria-prima (CABRAL et al., 2007), no entanto, outras espécies também são utilizadas em menor escala (KEINERT JR.; MATOS, 1987), e muitas ainda vêm sendo avaliadas quanto a sua viabilidade para inserção no processo produtivo e melhoria da qualidade destes painéis (TRIANOSKI, 2010).

O Acrocarpus fraxinifolius (Mundani), espécie originária das regiões tropicais de alta pluviosidade da Ásia (WHITMORE; OTAROLA, 1976), demonstrou em avaliação inicial, elevado potencial para produção de aglomerados, e de modo geral, propriedades tecnológicas superiores ao Pinus taeda, tradicionalmente utilizado na indústria de painéis no Brasil (TRIANOSKI, 2010). Características sobre a silvicultura, crescimento, produtividade volumétrica, propriedades tecnológicas da madeira e figuras relativas a esta espécie podem ser obtidas com maiores detalhes em Trianoski (2010).

Embora seja uma espécie com elevada taxa de crescimento (RAÍ, 1976) e candidata a produção de madeira de curta rotação (MAGHEMBE; PRINS, 1994), no Brasil, os plantios de Acrocarpus ainda são poucos, sendo necessários, desta forma, incentivos para a implantação de grandes áreas e máximo aproveitamento das toras disponíveis, as quais apresentam percentual de casca médio de $7,1 \%,(5,9$ a $10,2 \%)$, a partir de indivíduos com 8 anos de idade (PRADO et al., 2003).

$\mathrm{O}$ aproveitamento integral da madeira, característico da indústria de painéis aglomerados, tem utilizado subprodutos da indústria madeireira (costaneiras, rolos resto, serragem, maravalhas, etc.) e toras de pequeno diâmetro que não são utilizadas em serrarias e laminadoras (GALVÃO, 2000). Já a casca, um dos subprodutos gerados, foi, por muito tempo, desprezada durante a industrialização da madeira (PANSHIN; DE ZEEW, 1980) sendo atualmente, utilizada para a geração de energia. Teixeira (2003) ressalta que entre outros, este subproduto não tem sido aproveitado corretamente devido ao desconhecimento ou à inexistência de tecnologias disponíveis a serem prontamente transferidas.

De acordo com Iwakiri et al. (2005), a remoção da casca é primordial para a fabricação de aglomerados, tendo em vista sua influência negativa sobre as propriedades dos painéis. Vignote e Jiménez (1996) complementam que a presença de casca nos painéis diminui a qualidade da superfície, bem como a resistência, reduzindo sua aceitação e valor de mercado. Outro aspecto negativo relativo à casca, diz respeito à quantidade, em que determinadas espécies podem apresentar de 10 a $15 \%$ do volume das toras, acarretando problemas de estocagem e remoção, além de problemas ambientais (IWAKIRI et al., 2005).

Por outro lado, Maloney (1993) relata que a casca pode e tem sido usada em pequenas quantidades para a produção de aglomerados. Brito et al., (2005) avaliando as propriedades físicas e mecânicas de painéis aglomerados de Pinus elliottii com adição de casca de Eucalyptus pellita, concluíram que a adição de até $10 \%$ de casca é tecnologicamente viável na produção de aglomerados convencionais. Dost (1971) citado por Moslemi (1974), afirma que o crescente acréscimo de casca em até $30 \%$ reduziu consideravelmente a absorção de água de painéis aglomerados produzidos com Sequoia sempervirens (Redwood), no entanto, o inchamento em espessura foi totalmente prejudicado.

Tendo em vista a possibilidade de utilização de casca na produção de painéis aglomerados, a sua influência na qualidade final do produto, a contribuição ambiental gerada pela sua correta utilização em diferentes processos e a maximização do aproveitamento da matéria-prima, este trabalho teve como 
objetivo avaliar as propriedades físicas e mecânicas de painéis aglomerados de Acrocarpus fraxinifolius produzidos com diferentes percentuais de casca.

\section{MATERIAL E MÉTODO}

O Acrocarpus fraxinifolius utilizado neste trabalho foi proveniente de um plantio experimental com 19,5 anos de idade, localizado em Corupá-SC, de onde foram coletadas 5 árvores para o desenvolvimento do plano experimental. As árvores apresentaram altura total média de $22,74 \mathrm{~m}$, altura comercial média de $15,98 \mathrm{~m}$, DAP médio de $21,68 \mathrm{~cm}$ e volume médio com casca e sem casca de $0,26 \mathrm{~m}^{3} \mathrm{e}$ $0,25 \mathrm{~m}^{3}$, respectivamente.

O percentual de casca foi determinado a partir da diferença entre os volumes com e sem casca, conforme recomendado por Machado e Figueiredo Filho (2006). A massa específica básica da madeira foi determinada a partir de discos retirados a cada $0,40 \mathrm{~m}$ ao longo do fuste e de acordo com procedimentos recomendados pela Norma COPANT 461/1972. A massa específica básica da casca foi obtida a partir de medições do volume em volumenômetro de mercúrio e peso seco em balança analítica. A massa específica das composições foi determinada de acordo com Moslemi (1974).

$\mathrm{O}$ restante do material foi processado em laboratório, em picador de disco para geração de cavacos, com dimensões nominais de $25 \mathrm{~mm}$ de comprimento, $0,7 \mathrm{~mm}$ de espessura e largura variável. O Pinus taeda, utilizado para fins comparativos foi coletado na forma de partículas para camada interna em processo industrial de painéis aglomerados. Após a obtenção dos cavacos e da casca, foram amostradas e preparadas quantidades representativas de material para determinação do $\mathrm{pH}$, conforme normas TAPPI 257:2002, TAPPI 264:1997 e 252:2002, respectivamente.

Após a secagem dos cavacos e da casca do Acrocarpus, todo o material foi reprocessado em moinho de martelo, para obtenção das partículas tipo sliver. As partículas e a casca foram classificadas em peneiras com granulometrias de 8 e 30 mesh. Após esta classificação, foi efetuada a secagem final, obtendo-se um teor de umidade próximo a 3\%.

Os painéis foram produzidos de acordo com um delineamento inteiramente casualizado com duas repetições (painéis) por tratamento, conforme Tabela 1.

Para a produção dos painéis foi utilizada resina ureia-formaldeído, doada pela empresa Arauco do Brasil, a qual possuía teor de sólidos de $63,9 \%$, viscosidade de $668 \mathrm{cP}, \mathrm{pH}$ de 8,57 , densidade de $1,28 \mathrm{~g} / \mathrm{cm}^{3}$ e tempo de gelatinização de 1,06 min, e, $1 \%$ de parafina. A resina foi aplicada na proporção de $8 \%$ de sólidos, base peso seco de partículas.

Os painéis foram manufaturados de forma homogênea com dimensões de 50 x 50 x $1,5 \mathrm{~cm}$, massa específica nominal de $0,75 \mathrm{~g} / \mathrm{cm}^{3}$, e consolidados a uma temperatura de $160^{\circ} \mathrm{C}$, pressão específica de $40 \mathrm{kgf} / \mathrm{cm}^{2}$ e tempo de 8 minutos. Após a manufatura dos painéis, os mesmos foram conduzidos à câmara de climatização com condições ambientais controladas $\left(20 \pm 2^{\circ} \mathrm{C}\right.$ e $65 \pm 5 \%$ UR), até atingirem umidade de equilíbrio.

A qualidade dos painéis foi avaliada de acordo com a metodologia proposta pela Norma Europeia e pela Norma Brasileira. As propriedades físicas determinadas foram: massa específica aparente (EN 323:2002) e absorção de água e inchamento em espessura após 2 e 24 horas (EN 317:2002). As propriedades mecânicas resumiram-se em flexão estática (EN 310:2002), tração perpendicular à superfície (EN 319:2002) e resistência ao arrancamento de parafuso (NBR 14810$3: 2006)$. Os valores experimentais foram comparados com os requisitos propostos pela Norma EN 312:2003, NBR 14810-2:2006 e com os valores obtidos a partir do tratamento de referência (T1 100\% Pinus taeda).

TABELA 1: Delineamento experimental.

TABLE 1: Experimental design.

\begin{tabular}{cc}
\hline Tratamento & Composição \\
\hline 1 & $100 \%$ Pinus taeda \\
2 & $100 \%$ Acrocarpus fraxinifolius \\
3 & $90 \%$ Acrocarpus fraxinifolius $-10 \%$ Casca de Acrocarpus fraxinifolius \\
4 & $80 \%$ Acrocarpus fraxinifolius $-20 \%$ Casca de Acrocarpus fraxinifolius \\
5 & $70 \%$ Acrocarpus fraxinifolius $-30 \%$ Casca de Acrocarpus fraxinifolius \\
\hline
\end{tabular}


Os dados obtidos foram submetidos à análise estatística ( $95 \%$ de probabilidade) por meio dos testes de Grubbs, Shapiro Wilks, Bartlett, Análise de Variância, e Comparação de Média de Tukey. Para os dados de Absorção de Água e Inchamento em Espessura após 2 e 24 horas, houve a necessidade de transformação de dados por função do tipo raiz quadrada para atendimento dos requisitos à distribuição normal.

\section{RESULTADOS E DISCUSSÃO}

\section{Propriedades da madeira}

O percentual médio de casca para o Acrocarpus fraxinifolius foi de $6,78 \%$ com coeficiente de variação de $1,12 \%$. Em comparação com as informações disponíveis em literatura, como por exemplo, Foelkel (2009) que relata que o teor de casca em florestas comerciais de Eucalyptus varia entre $10 \%$ e $18 \%$, em florestas clonais geneticamente melhoradas este percentual é de $9 \%$ a $12 \%$ e em outras espécies pode chegar até 30\%; Vital et al. (1989) que obtiveram percentuais de casca de 7,90\% a 11,82 para Eucalyptus grandis; Costa e Rezende (2008) que encontraram valores entre 6,3\% e 10,1\% para reflorestamento seminal, clones e híbridos de Eucalyptus grandis e Eucalyptus urophylla; Colpini et al. (2009) que encontraram valor médio de 7,45\% para 47 espécies de interesse comercial e potencial da Floresta Ombrófila Aberta no Noroeste do Mato Grosso; e Haselein et al. (2004) que determinaram o potencial volumétrico da casca em árvores matrizes de Eucalyptus grandis de 3,46\%, é possível afirmar que a espécie em estudo apresenta um percentual de casca que pode ser considerado de médio a baixo, contribuindo para questões relativas à minimização de resíduos gerados, otimização do transporte e comercialização. Já em relação às espécies de Pinus, as quais são as mais utilizadas para a produção de painéis aglomerados, nota-se que Trianoski (2012) obteve percentuais de casca de $11,81 \%$ para o Pinus caribaea var. bahamensis; $11,72 \%$ para o Pinus caribaea var. caribaea; $14,09 \%$ para o Pinus caribaea var. hondurensis; $8,78 \%$ para o Pinus chiapensis; $14,16 \%$ para o Pinus maximinoi; 9,63\% para o Pinus oocarpa; $10,35 \%$ para o Pinus tecunumanii e $10,45 \%$ para o Pinus taeda, sendo possível observar que o Acrocarpus apresentou valor médio inferior a todas às espécies citadas, indicando com isso uma vantagem frente a esta característica.
Os resultados inerentes às propriedades de massa específica básica e $\mathrm{pH}$ das madeiras utilizadas e da casca são apresentados na Tabela 2.

TABELA 2: Valores médios da massa específica e $\mathrm{pH}$ da madeira, da casca e composições.

TABLE 2: Average values of specific gravity and the $\mathrm{pH}$ of wood, bark and mixtures.

\begin{tabular}{ccc}
\hline Tratamento & $\begin{array}{c}\text { Massa específica básica* } \\
\left(\mathrm{g} / \mathrm{cm}^{3}\right)\end{array}$ & $\mathrm{pH}$ \\
\hline $100 \% \mathrm{Pt}$ & 0,450 & $4,56 \mathrm{e}$ \\
$100 \%$ Af & 0,458 & $5,14 \mathrm{a}$ \\
$90 \%$ Af $-10 \%$ casca & 0,466 & $5,04 \mathrm{~b}$ \\
$80 \%$ Af $-20 \%$ casca & 0,473 & $4,93 \mathrm{c}$ \\
$70 \%$ Af $-30 \%$ casca & 0,481 & $4,91 \mathrm{c}$ \\
Casca & 0,534 & $4,66 \mathrm{~d}$ \\
\hline
\end{tabular}

Em que: $\mathrm{Pt}=$ Pinus taeda $; \mathrm{Af}=$ Acrocarpus fraxinifolius; Médias seguidas de uma mesma letra em uma mesma coluna são estatisticamente iguais pelo Teste de Tukey a $95 \%$ de probabilidade.

Verifica-se que a massa específica básica, tanto das madeiras de Pinus e de Acrocarpus, quanto às misturas entre a madeira de Acrocarpus e os diferentes percentuais de casca apresentaram-se adequadas à faixa nominal recomendada para a produção de painéis aglomerados, conforme proposto por Maloney (1993). Observou-se que os tratamentos apresentaram potencial hidrogeniônico dentro do intervalo de 3,0 e 5,5 descrito por Stamm (1964). O maior grau de acidez foi apresentado pelo tratamento 1, composto pela espécie Pinus taeda, a qual apresentou valor similar a estudos recentes realizados por Prata (2010) e Trianoski (2010; 2012). O Acrocarpus fraxinifolius, demonstrou o maior valor de $\mathrm{pH}$, e à medida que se aumentou o percentual de casca na composição, o pH reduziu gradativamente, tornando-se mais ácido. Este fato é justificado segundo os relatos de Vital et al. (1989) e Foelkel (2009) que afirmam que o teor de extrativos da casca é sensivelmente mais elevado do que aquele encontrado na madeira. Assim, o maior teor de extrativos normalmente contribui para um $\mathrm{pH}$ mais ácido.

\section{Propriedades físicas dos painéis}

Na Tabela 3 são apresentadas as médias da massa específica aparente e da razão de compactação dos tratamentos propostos. 
TABELA 3: Valores médios de massa específica aparente e da razão de compactação.

TABLE 3: Average values of the board density and the compaction ratio.

\begin{tabular}{ccc}
\hline Tratamento & $\begin{array}{c}\text { Massa específica } \\
\left(\mathrm{g} / \mathrm{cm}^{3}\right)\end{array}$ & $\begin{array}{c}\text { Razão de } \\
\text { Compactação }\end{array}$ \\
\hline \multirow{2}{*}{$100 \% \mathrm{Pt}$} & $0,680 \mathrm{a}$ & $1,51 \mathrm{a}$ \\
& $(4,80)$ & $(4,86)$ \\
$100 \% \mathrm{Af}$ & $0,695 \mathrm{a}$ & $1,48 \mathrm{a}$ \\
& $(6,76)$ & $(4,35)$ \\
$90 \%$ Af -10\% casca & $0,705 \mathrm{a}$ & $1,49 \mathrm{a}$ \\
& $(5,33)$ & $(6,61)$ \\
$80 \%$ Af $-20 \%$ casca & $0,689 \mathrm{a}$ & $1,48 \mathrm{a}$ \\
& $(5,89)$ & $(7,14)$ \\
$70 \%$ Af $-30 \%$ casca & $0,694 \mathrm{a}$ & $1,47 \mathrm{a}$ \\
& $(5,95)$ & $(7,81)$ \\
\hline
\end{tabular}

Em que: $\mathrm{Pt}=$ Pinus taeda Af = Acrocarpus fraxinifolius; Médias seguidas de uma mesma letra em uma mesma coluna são estatisticamente iguais pelo Teste de Tukey a $95 \%$ de probabilidade. Valores entre parentes referem-se ao Coeficiente de variação.

Observa-se pela Análise de Variância que todos os tratamentos apresentaram valores médios de massa específica aparente estatisticamente iguais entre si, dispensando a aplicação da Análise de Covariância para as demais propriedades obtidas. Em relação à razão de compactação, nota-se que os valores foram superiores a 1,3, conforme preconizado por Moslemi (1974) e Maloney (1993), indicando que houve contato suficiente entre as partículas.

Os resultados referentes à absorção de água e inchamento em espessura são apresentados na Tabela 4.

Para a absorção de água após 2 e 24 horas de imersão, os valores variaram entre $7,36 \%$ a $26,66 \%$ e $20,58 \%$ a $64,07 \%$, respectivamente, denotando diferença estatística entre os tratamentos propostos. Observa-se, que com o aumento do percentual de casca adicionado, houve uma tendência de redução de absorção de água para ambas as condições de ensaio. Resultados similares foram obtidos por Dost (1971) citado por Moslemi (1974) que relatou que painéis produzidos com Sequoia sempervirens, apresentaram uma redução considerável na absorção de água com o acréscimo de até $30 \%$ de casca.

Comparados com a testemunha, nota-se que nenhum dos tratamentos apresentou menor índice de absorção, onde estes resultados podem estar associados à geometria das partículas, as quais foram obtidas em processo industrial. Por outro lado, quan- do comparados com os resultados disponíveis em literatura, verifica-se que os resultados obtidos nesta pesquisa foram menores do que os encontrados por Brito et al. (2005) que, avaliando painéis aglomerados de uma camada de Pinus elliottii com cascas de Eucalyptus pellita, obtiveram valores de 95\% a $127 \%$ após 2 horas de imersão e $106 \%$ a $138 \%$ após 24 horas de imersão, e de Batista et al. (2007) que encontraram valores entre $134,1 \%$ a $167,5 \%$ ( 2 horas) e $143,7 \%$ a $183,8 \%$ (24 horas) para painéis de três camadas, confeccionados também, com Pinus elliottii e cascas de Eucalyptus pellita.

Os valores médios de inchamento em espessura variaram entre $3,6 \%$ a $7,9 \%$ após 2 horas e entre $8,5 \%$ a $19,5 \%$ após 24 horas. Foi constatada diferença estatística significativa entre os tratamentos propostos, onde o tratamento 5 , com $30 \%$ de casca foi estatisticamente inferior aos demais tratamentos manufaturados com Acrocarpus e casca, no entanto, nenhum deles apresentou maior estabilidade dimensional quando comparado com os painéis de Pinus taeda.

Em comparação com os resultados apresentados em literatura, verifica-se que o inchamento em espessura obtido a partir desta pesquisa foi inferior aos resultados encontrados por pesquisadores como Naumann et al. (2008) que obtiveram valores entre $11,5 \%$ a $20,2 \%$ ( 2 horas) e $14,2 \%$ a $23,3 \%$ (24 horas) para painéis aglomerados produzidos com Eucalyptus urophylla e Schizolobium amazonicum,

TABELA 4: Valores médios da absorção de água e inchamento em espessura.

TABLE 4: Average values of water absorption and thickness swelling.

\begin{tabular}{ccccc}
\hline Tratamento & $\begin{array}{c}\text { AA 2h } \\
(\%)\end{array}$ & $\begin{array}{c}\text { AA 24h } \\
(\%)\end{array}$ & $\begin{array}{c}\text { IE 2h } \\
(\%)\end{array}$ & $\begin{array}{c}\text { IE 24h } \\
(\%)\end{array}$ \\
\hline \multirow{2}{*}{$100 \%$ Pt } & $7,36 \mathrm{c}$ & $20,58 \mathrm{c}$ & $3,61 \mathrm{c}$ & $8,53 \mathrm{c}$ \\
& $(24,65)$ & $(13,42)$ & $(21,54)$ & $(12,60)$ \\
$100 \%$ Af & $26,66 \mathrm{a}$ & $64,07 \mathrm{a}$ & $6,84 \mathrm{a}$ & $18,85 \mathrm{a}$ \\
& $(23,18)$ & $(9,17)$ & $(22,57)$ & $(13,06)$ \\
$90 \%$ Af -10\% casca & $24,84 \mathrm{a}$ & $62,73 \mathrm{a}$ & $7,89 \mathrm{a}$ & $19,46 \mathrm{a}$ \\
& $(24,84)$ & $(12,01)$ & $(28,57)$ & $(15,95)$ \\
$80 \%$ Af -20\% casca & $23,56 \mathrm{ab}$ & $58,00 \mathrm{a}$ & $6,42 \mathrm{a}$ & $17,61 \mathrm{a}$ \\
& $(28,11)$ & $(12,19)$ & $(21,63)$ & $(10,83)$ \\
$70 \%$ Af -30\% casca & $19,70 \mathrm{~b}$ & $49,60 \mathrm{~b}$ & $4,91 \mathrm{~b}$ & $12,94 \mathrm{~b}$ \\
& $(24,26)$ & $(17,65)$ & $(27,91)$ & $(18,17)$ \\
\hline
\end{tabular}

Em que: $\mathrm{Pt}=$ Pinus taeda $; \mathrm{Af}=$ Acrocarpus fraxinifolius; Médias seguidas de uma mesma letra em uma mesma coluna são estatisticamente iguais pelo Teste de Tukey a 95\% de probabilidade. Valores entre parentes referem-se ao Coeficiente de variação. 
e por Iwakiri et al. (2010) que encontraram valores entre $24,0 \%$ e $31,34 \%$ para painéis produzidos com Pinus taeda, Pinus caribaea var. caribaea e Pinus caribaea var. bahamensis.

De maneira geral, é possível constatar que o aumento do percentual de casca contribuiu para a melhoria das propriedades de estabilidade dimensional, onde este resultado pode estar associado à estrutura anatômica e à composição química da casca. Segundo Foelkel (2009), as cascas são péssimas matérias-primas fibrosas e com maior percentual de minerais, o que proporciona uma menor absorção e menor inchamento. Apresentam ainda, teor de extrativos superior à madeira (VITAL et al., 1989), os quais podem promover maior inibição nas propriedades de absorção de água e inchamento em espessura.

\section{Propriedades mecânicas dos painéis}

$\mathrm{Na}$ Tabela 5 são apresentados os valores médios de flexão estática, tração perpendicular às faces da placa e resistência ao arrancamento de parafuso na superfície e topo.

Os valores médios do módulo de ruptura variaram de $12,88 \mathrm{MPa}$ a $13,09 \mathrm{MPa}$, não sendo constatada diferença estatística significativa entre os tratamentos propostos. Para o módulo de elasticidade, verifica-se que a variação foi de $1.832,30 \mathrm{MPa}$ a 1.948,22 $\mathrm{MPa}$, onde os valores médios também foram estatisticamente iguais entre si. Estes resultados permitem afirmar que, para as condições des- critas e executadas durante o desenvolvimento do plano experimental, as matérias-primas apresentam a mesma qualidade e que o crescente aumento de casca, ao invés de interferir negativamente no módulo de ruptura e o módulo de elasticidade, conforme relatado por Moslemi (1974), manteve as propriedades estáveis.

Em relação aos requisitos normativos preconizados pela norma EN 312:2003, pode-se dizer que todos os tratamentos apresentaram a resistência mínima de $13 \mathrm{MPa}$ e $1.600 \mathrm{MPa}$ para módulos de ruptura e elasticidade, respectivamente. Em comparação com os resultados descritos em literatura e sob as mesmas condições, observa-se que os valores médios obtidos nesta pesquisa foram similares aos encontrados por Iwakiri et al. (2010) que obtiveram valores entre $12,03 \mathrm{MPa}$ a $18,08 \mathrm{MPa}$ para módulo de ruptura e $1.886 \mathrm{MPa}$ a $2.515 \mathrm{MPa}$ para módulo de elasticidade, a partir de painéis produzidos com Pinus taeda, Pinus caribaea caribaea e Pinus caribaea bahamensis. Por outro lado, os resultados desta pesquisa foram superiores aos obtidos por Brito et al. (2005) para misturas de madeiras de Pinus elliottii com 10, 20 e 30\% de casca de Eucalyptus pellita, cujos valores médios foram respectivamente de 10,56 MPa, 7,86 MPa e 8,49 MPa para o módulo de ruptura e de $1.704 \mathrm{MPa}, 1.648 \mathrm{MPa}$ e $1.635 \mathrm{MPa}$ para o módulo de elasticidade.

Para a propriedade de tração perpendicular, os valores médios variaram de $0,86 \mathrm{MPa}$ a $1,12 \mathrm{MPa}$, sendo constatada diferença estatística significativa entre os tratamentos manufaturados com Pinus

TABELA 5: Valores médios das propriedades mecânicas.

TABLE 5: Average values of the mechanical properties.

\begin{tabular}{|c|c|c|c|c|c|}
\hline \multirow[b]{2}{*}{ Tratamento } & \multicolumn{2}{|c|}{ Flexão estática } & \multirow[b]{2}{*}{$\begin{array}{c}\text { Tração Perpendicular } \\
(\mathrm{MPa})\end{array}$} & \multicolumn{2}{|c|}{ Arrancamento de Parafuso } \\
\hline & $\begin{array}{l}\text { MOR } \\
(\mathrm{MPa})\end{array}$ & $\begin{array}{l}\mathrm{MOE} \\
(\mathrm{MPa})\end{array}$ & & $\begin{array}{l}\text { Superfície } \\
\text { (N) }\end{array}$ & $\begin{array}{c}\text { Topo } \\
\text { (N) }\end{array}$ \\
\hline $100 \% \mathrm{Pt}$ & $\begin{array}{l}12,88 \mathrm{a} \\
(12,89)\end{array}$ & $\begin{array}{c}1.948,22 \mathrm{a} \\
(13,03)\end{array}$ & $\begin{array}{c}0,86 \mathrm{~b} \\
(12,58)\end{array}$ & $\begin{array}{c}1.031,86 \mathrm{~b} \\
(14,94)\end{array}$ & $\begin{array}{c}846,06 \mathrm{~b} \\
(16,11)\end{array}$ \\
\hline $100 \%$ Af & $\begin{array}{l}13,09 \mathrm{a} \\
(11,64)\end{array}$ & $\begin{array}{c}1.861,25 \mathrm{a} \\
(8,65)\end{array}$ & $\begin{array}{c}1,12 \mathrm{a} \\
(10,75)\end{array}$ & $\begin{array}{c}1.482,96 \mathrm{a} \\
(11,76)\end{array}$ & $\begin{array}{c}1.088,18 \mathrm{ab} \\
(15,36)\end{array}$ \\
\hline $90 \%$ Af $-10 \%$ casca & $\begin{array}{l}13,00 \mathrm{a} \\
(15,29)\end{array}$ & $\begin{array}{c}1.832,30 \mathrm{a} \\
(16,02)\end{array}$ & $\begin{array}{l}0,97 \mathrm{ab} \\
(18,00)\end{array}$ & $\begin{array}{c}1.360,38 \mathrm{a} \\
(17,37)\end{array}$ & $\begin{array}{c}1.126,10 \mathrm{a} \\
(24,09)\end{array}$ \\
\hline $80 \%$ Af $-20 \%$ casca & $\begin{array}{c}13,00 \mathrm{a} \\
(9,27)\end{array}$ & $\begin{array}{c}1.926,90 \mathrm{a} \\
(9,88)\end{array}$ & $\begin{array}{l}1,02 \mathrm{ab} \\
(17,73)\end{array}$ & $\begin{array}{l}1.325,76 \mathrm{a} \\
(18,57)\end{array}$ & $\begin{array}{c}1.052,15 \mathrm{ab} \\
(14,30)\end{array}$ \\
\hline $70 \%$ Af $-30 \%$ casca & $\begin{array}{l}13,01 \mathrm{a} \\
(10,38)\end{array}$ & $\begin{array}{c}1.927,04 \mathrm{a} \\
(7,60)\end{array}$ & $\begin{array}{l}1,01 \mathrm{ab} \\
(24,08)\end{array}$ & $\begin{array}{c}1.275,65 \mathrm{ab} \\
(19,04)\end{array}$ & $\begin{array}{c}1.155,62 \mathrm{a} \\
(21,84)\end{array}$ \\
\hline
\end{tabular}

Em que: $\mathrm{Pt}=$ Pinus taeda; Af = Acrocarpus fraxinifolius; Médias seguidas de uma mesma letra em uma mesma coluna são estatisticamente iguais pelo Teste de Tukey a 95\% de probabilidade. Valores entre parentes referem-se ao Coeficiente de variação. 
taeda e Acrocarpus fraxinifolius. Observa-se também, que os painéis produzidos com $100 \%$ de partículas de Acrocarpus, e com madeira de Acrocarpus e diferentes percentuais de casca, foram estatisticamente iguais entre si, e que a adição de casca não afetou a adesão entre as partículas, conforme preconizado em literatura.

Em referência ao requisito mínimo de 0,35 MPa estabelecido pela norma EN 312:2003, nota-se que todos os tratamentos apresentaram valores adequados. Considerando os resultados encontrados por outros pesquisadores, verifica-se que os valores médios obtidos neste trabalho foram superiores aos descritos por Dacosta et al. (2005) que avaliando painéis aglomerados produzidos com resíduos do processamento mecânico de Pinus elliotti encontraram valores entre $0,09 \mathrm{Mpa}$ a $0,23 \mathrm{MPa}\left(0,92\right.$ a $\left.2,36 \mathrm{kgf} / \mathrm{cm}^{2}\right)$ e por Melo e Del Menezzi (2010) que obtiveram valores entre $0,28 \mathrm{MPa}$ a 0,35 MPa para painéis produzidos com Eucalyptus grandis.

Os resultados obtidos para resistência ao arrancamento de parafuso apresentaram valores médios no intervalo de $1.031,86 \mathrm{~N}$ a $1.482,96 \mathrm{~N}$ para o ensaio de superfície e $846,06 \mathrm{~N}$ a $1.155,62 \mathrm{~N}$ para o ensaio de topo. Observa-se, que para esta propriedade, a adição de casca também não exerceu influência negativa sobre a resistência. Notase também, que todos os tratamentos apresentaram para ambas as faces ensaiadas, valores médios absolutos superiores ao tratamento utilizado como testemunha, constituído pelo Pinus taeda.

Considerando o atendimento ao requisito normativo, todos os tratamentos apresentaram valores médios superiores ao prescrito pela norma NBR 18810-3:2006, que preconiza uma resistência mínima de $1.020 \mathrm{~N}$ para o ensaio de superfície e $800 \mathrm{~N}$ para o ensaio de topo. Em relação aos resultados relatados em outros estudos, verificam-se valores superiores aos determinados por Dacosta et al. (2005), com variação de $456,11 \mathrm{~N}$ a $745,31 \mathrm{~N}$ (46kgf a 76kgf), para painéis manufaturados com resíduos do processamento mecânico de Pinus elliotti, e por outro lado, resultados similares aos obtidos por Trianoski (2010) em avaliação de espécies alternativas de rápido crescimento para produção de painéis aglomerados.

\section{CONCLUSÕES}

Os painéis produzidos com partículas de madeira de Acrocarpus fraxinifolius apresentaram viabilidade técnica para a produção de painéis de madeira aglomerada.

Os painéis produzidos com a adição de casca apresentaram propriedades mecânicas superiores aos requisitos mínimos estabelecidos pelas normas EN 312:2003 e NBR 14.810-2:2006.

A crescente adição da casca aparentemente não afetou negativamente as propriedades físicas e mecânicas, como normalmente é esperado. $\mathrm{O}$ processamento integral de toras de Acrocarpus fraxinifolius demonstra-se tecnicamente viável, tendo em vista o percentual de $6,78 \%$ de casca encontrado, a partir das árvores amostradas.

É possível a produção de painéis aglomerados de Acrocarpus fraxinifolius (com até 30\% de casca) nas condições desse estudo, agregando desta forma, maior valor à espécie.

\section{AGRADECIMENTOS}

Os autores expressam seus agradecimentos a Battistella Florestal pelo uso do plantio experimental de Acrocarpus fraxinifolius e à Arauco do Brasil pela doação das partículas de Pinus taeda, resina e parafina.

\section{REFERÊNCIAS BIBLIOGRÁFICAS}

ASSOCIAÇÃO BRASILEIRA DE NORMAS TÉCNICAS (ABNT). NBR 14810 - 2 - Chapas de madeira aglomerada - Parte 2 - Requisitos. 2006. ASSOCIAÇÃO BRASILEIRA DE NORMAS TÉCNICAS (ABNT). NBR 14810 - 3 - Chapas de madeira aglomerada - Parte 3 - Métodos de Ensaio. 2006.

BATISTA, D. C. et al. Fabricação de aglomerados de três camadas com madeira de Pinus elliottii Engelm e casca de Eucalyptus pellita F. Muell. Cerne, Lavras, v. 13, n. 2, p. 178-187, 2007.

BRITO, E. O. et al. Chapas de madeira aglomerada de uma camada de Pinus elliottii Engelm com a adição das cascas de Eucalyptus pellita F. Muell. Cerne, Lavras, v. 11, n. 4, p. 369-375, 2005.

CABRAL, C. P. et al. Propriedades de chapas de aglomerado confeccionadas com misturas de partículas de Pinus spp e Pinus elliottii. Revista Árvore, Viçosa, v. 31, n. 5, p. 897-905, 2007. COLPINI, C. et al. Determinação do volume, fator de forma e da porcentagem da casca de árvores individuais em uma Floresta Ombrófila Aberta na Região Noroeste de Mato Grosso. Acta Amazônica, Manaus, v. 39, n. 1, p. 97-104, 2009. 
COMISSÃO PANAMERICANA DE NORMAS TÉCNICAS. COPANT 461. Determinación del peso especifico aparente. 1972.

COSTA, V. E.; REZENDE, M. A. Produtividade de reflorestamentos de seminal, clone e híbrido de Eucalyptus grandis x E. urophylla na região de Botucatu, SP. Revista Energia na Agricultura, Botucatu v. 23, n. 2, p. 115-128, 2008.

DACOSTA, L. P. E. et al. Qualidade das chapas de partículas aglomeradas fabricadas com resíduos do processamento mecânico de Pinus elliottii (Engelm). Ciência Florestal, Santa Maria, v. 15, n. 3, p. 311-322, 2005.

EUROPEAN STANDARD. EN 310. Determination of modulus of elasticity in bending and of bending strength. Versão portuguesa, 2002.

EUROPEAN STANDARD. EN 312. Particleboards - Specifications. .English version. 2003.

EUROPEAN STANDARD. EN 317. Determination of swelling in thickness after immersion in water. English version. 2002.

EUROPEAN STANDARD. EN 319. Determinação da resistencia à tração perpendicular às faces da placa. Versão Portuguesa. 2002.

EUROPEAN STANDARD. EN 323. Determinação da massa volúmica. Versão Portuguesa. 2002.

FOELKEL, C. Casca da árvore do eucalipto: Aspectos morfológicos, fisiológicos, florestais, ecológicos, e industriais visando a produção de celulose e papel. Eucalyptus Book Online e Newsletter 2009. Disponível em: <http://www. eucalyptus.com.br/capitulos/capitulo_casca.pdf > Acesso em 22/07/2012.

GALVÃO, A. P. M. Reflorestamento de propriedades rurais para fins produtivos $e$ ambientais. Brasília : EMBRAPA, 2000. 351 p.

HASELEIN, C. R. et al.Características tecnológicas da madeira de árvores matrizes de Eucalyptus grandis. Ciência Florestal, Santa Maria, v. 14, n. 2, p. 145-155, 2004.

IWAKIRI, S. et al. Use of Wood from Pinus caribaea var. caribaea and Pinus caribaea var. bahamensis for production of particleboard panel. Cerne, Lavras, v. 16, n. 2, p. 193-198, 2010.

IWAKIRI, S. et al. Painéis de madeira aglomerada. In: Painéis de madeira reconstituída. Curitiba, Fupef, 2005, p. 123-158.

IWAKIRI, S. et al. Utilização de resíduos de serrarias na produção de chapas de madeira aglomerada de Eucalyptus saligna, Eucalyptus citriodora e Eucalyptus pilularis. Floresta e Ambiente, Seropédica, v. 7, n.1, p. 251-256, 2000.
KEINERT JR. S.; MATOS, J. L. M. Utilização de Pinus pinaster para fabricação de chapas de partículas. Floresta, Curitiba, v. 17, n. 1-2, p. 113-120, 1987.

MACHADO, S. A.; FIGUEIREDO FILHO, A. Dendrometria. 2. ed. Guarapuava: Unicentro, 2006. $316 \mathrm{p}$.

MAGHEMBE, J. A.; PRINS, H. Performance of multipurpose trees for agroforestry two years after planting at Makoka, Malawi. Forest Ecology and Management, Amsterdam, v. 64, n. 2-3, p. 171-182, 1994.

MALONEY, T. M. Modern particleboard and dryprocess fiberboard manufacturing. San Francisco: M. Freeman, 1993. 689 p.

MELO, R. R.; DEL MENEZZI, C. H. S. Influência da massa específica nas propriedades físico-mecânicas de painéis aglomerados. Silva Lusitana, Oeiras, v. 18, n.1, p.5-73, 2010.

MOSLEMI, A. A. Particleboard: Materials. London: Southern Illinois: University Press. 1974. $244 \mathrm{p}$.

NAUMANN. R. B. et al. Propriedades de chapas fabricadas com partículas de madeira de Eucalyptus urophylla S.T.Blake e Schizolobium amazonicum Herb. Àrvore, Viçosa, v. 32, n. 6, p. 1143-1150, 2008.

PANSHIN, A. J.; DE ZEEW, C. Textbook of wood technology. $4^{\text {th }}$ ed. New York: MC Graw Hill, 1980. $722 \mathrm{p}$.

PRADO, C. A. et al. Características físicas e químicas da madeira de Acrocarpus fraxinifolius Wight \& Arn. Colombo: Embrapa Florestas, 2003, 14 p. (Boletim de Pesquisa e Desenvolvimento n.14) PRATA, J. G. Estudo da viabilidade tecnológica do uso de espécies de Pinus tropicais para produção de painéis colados lateralmente (EDGE GLUED PANEL- EGP) 2010. 114 f. Tese (Doutorado em Engenharia Florestal) - Universidade Federal do Paraná, Curitiba, 2010.

RAÍ, S. N. Pré-treatment of Acrocarpus fraxinifolius seeds. Indian Forester, Dehradun, v. 102, n. 8, p. 488-491, 1976.

STAMM, A. J. Wood and cellulose science. New York: The Ronald Press Company, 1964. 549 p.

TECHNICAL ASSOCIATION OF THE PULP AND PAPER INDUSTRY. TAPPI 264. Preparation of wood for chemical analysis. Atlanta, 1997.

TECHNICALASSOCIATION OF THE PULP AND PAPER INDUSTRY. TAPPI 252. $\mathrm{pH}$ and electrical conductivity of hot water extracts of pulp, paper, and paperboard. Atlanta, 2002. 
TECHNICALASSOCIATION OF THE PULP AND PAPER INDUSTRY. 2002. TAPPI 257.Sampling and preparing wood for analysis. Atlanta. 2002.

TRIANOSKI, R. Avaliação do potencial de espécies florestais alternativas de rápido crescimento para produção de painéis de madeira aglomerada. 2010. 260 f. Dissertação (Mestrado em Ciências Florestais) - Universidade Federal do Paraná, Curitiba. 2010.

TRIANOSKI, R. Avaliação da qualidade da madeira de espécies de Pinus tropicais por meiode métodos convencionai e não-destrutivos. 2012.
553 f. Tese (Doutorado em Ciências Florestais) Universidade Federal do Paraná, Curitiba. 2012.

VIGNOTE, P. S.; JIMÉNEZ, P. F. J. Tecnologia de la madera. Madrid, Ministerio de Africultura, Pesca y Alimentación, 1996, 602 p.

VITAL, B. R. et al. Influência da casca no rendimento e na qualidade do carvão de Eucalyptus grandis. IPEF, Piracicaba, n. 41/42, p. 44-49, 1989.

WHITMORE, J. L; OTAROLA, T. Acrocarpus fraxinifolius Wight, especie de rápido crecimiento inicial, buena forma y madera de usos múltiples. Turrialba, San José, v. 2, p. 201-204, 1976. 